\title{
Factors Associated with Occurrence and Recovery of Nonambulatory Dairy Cows in the United States
}

\author{
A. L. Green, ${ }^{1,2}$ J. E. Lombard, L. P. Garber, B. A. Wagner, and G. W. Hill \\ USDA:APHIS:VS, Centers for Epidemiology and Animal Health, 2150 Centre Ave., Bldg. B, Fort Collins, CO 80526-8117
}

\begin{abstract}
The primary objective of this study was to compare characteristics of US dairy operations that had one or more nonambulatory cows (unable to rise for any period of time) (cases) with operations that had no nonambulatory cows (controls) during 2004. A secondary objective was to describe factors associated with recovery of the last nonambulatory cow on the operation during 2004. Case dairy operations $(\mathrm{n}=1,822)$ more often fed a total mixed ration [odds ratio $(\mathrm{OR})=2.0$; confidence interval (CI): 1.1-3.4], produced more than $9,090 \mathrm{~kg}$ of milk $(\mathrm{OR}=2.8$; CI: 1.8-4.5), and were more likely to be of medium to large herd size (100 or more head of adult cows, OR = 3.7; CI: 2.2-6.2) compared with control dairies $(n=151)$. Compared with operations where the predominant flooring surface on which lactating cows stood or walked in winter was pasture, operations where pasture was not the predominant surface were at increased risk of having nonambulatory cows ( $\mathrm{OR}=4.7$; CI: 2.2 10.2). Cows nonambulatory for less than $24 \mathrm{~h}$ were more likely to recover compared with cows nonambulatory for $24 \mathrm{~h}$ or more $(\mathrm{OR}=3.0$; CI: 2.0-4.4). Cows that received calcium, phosphorus, or potassium while nonambulatory were more likely to recover $(\mathrm{OR}=3.6$; CI: 2.1-6.1) than cattle that did not receive these treatments. Cattle that were not repositioned periodically were more likely to recover $(\mathrm{OR}=2.1$; CI: $1.4-3.1)$, as were cattle that were not treated by a veterinarian before becoming nonambulatory ( $\mathrm{OR}=1.9$; CI: 1.1-3.3). These findings are consistent with prolonged recumbency and prior history of health issues, respectively. Nonambulatory cattle with hypocalcemia were more likely to recover $(\mathrm{OR}=6.0$; CI: 3.4-10.7) compared with nonambulatory cows with all other causes of a nonambulatory condition (analyzed collectively as a single variable but including cancer, clinical mastitis, diges-
\end{abstract}

Received November 16, 2007.

Accepted February 23, 2008.

${ }^{1}$ Corresponding author: alice.l.green@gmail.com

${ }^{2}$ Current address: Tennessee Department of Health, Communicable and Environmental Disease Services, Cordell Hull Building, 425 5th Avenue North, Nashville 37243. tive conditions, metabolic imbalances, neurological problems, respiratory disease, other, unknown). The results of this study reveal that the majority of US dairy operations have at least one nonambulatory dairy cow over the course of a year. Additionally, individual animal factors associated with being nonambulatory may lead to improved identification and treatment of animals that are nonambulatory for a prolonged period. From the perspective of recovery, considering euthanasia is appropriate for cows that have been nonambulatory for more than $24 \mathrm{~h}$.

Key words: dairy cattle, nonambulatory

\section{INTRODUCTION}

Nonambulatory cattle are important as a lost source of income and as one of the targeted cattle groups for bovine spongiform encephalopathy surveillance efforts. An estimated 270,000 cattle weighing $227 \mathrm{~kg}$ or more became nonambulatory on-farm in the United States in 2004. Of these animals, $155,000(57.4 \%)$ were dairy cattle and $85,000(31.5 \%)$ were beef cattle; the remainder were on mixed or other types of operations (National Agricultural Statistics Service; NASS, 2005). As a percentage of cattle inventory $227 \mathrm{~kg}$ or more, $1.2 \%$ of dairy cattle and $0.2 \%$ of beef cattle became nonambulatory in 2004 (calculated from NASS, 2006). Several studies have estimated the percentage of dairy operations that have nonambulatory cattle. The NASS study (NASS, 2005 ) reported that $26.5 \%$ of the operations had one or more nonambulatory cows in 2004. A second national study conducted by the National Animal Health Monitoring System (NAHMS) estimated that $78.2 \%$ of operations in 21 dairy states had one or more nonambulatory cows in 2004 (Green et al., 2006). The study reported here is a component of the NAHMS study. The latter estimate is similar to that of an Indiana study on dairy operations in which $76 \%$ of operations had one or more nonambulatory cows in 2004 (Dieterlen and Strasser, 2006). This wide range in farm-level estimates, which may be due in part to differences in the populations being sampled and with the definition of nonambulatory that was presented to the producer at the time of the interview, accentuates the need for a better under- 
standing of nonambulatory cattle problems in US dairy operations.

At the time this study was initiated, the United States did not have a specific definition for nonambulatory cattle. The current definition of nonambulatory cattle utilized by the United Kingdom ("unable to rise") and Canada ("unable to stand without assistance or to move without being dragged or carried") have no parameters for time or outcome (Defra, 2003; Government of Canada, 2004). To provide a complete picture of the occurrence and recovery of nonambulatory dairy cattle, and to be consistent with international standards, the NAHMS Nonambulatory Livestock study (this study) was designed to include nonambulatory cattle that were unable to stand or walk without assistance for any length of time, including those that recovered.

The objectives of this study were to compare herdlevel characteristics of US dairies that had one or more nonambulatory cows with dairies that had no nonambulatory cows during 2004 and to describe factors associated with recovery of individual nonambulatory cows.

\section{MATERIALS AND METHODS}

\section{Study Design and Population}

The Farm Security and Rural Investment Act of 2002 (also known as the 2002 Farm Bill) requested that the USDA investigate the scope, causes, and handling practices of nonambulatory livestock in the United States. Questions on the number and outcome of nonambulatory cattle were included in the NASS January 1, 2004, and January 1,2005 , cattle surveys obtaining responses from 50,000 respondents with cattle. The resultant data provided information upon which to design the study in 2005. As a follow-up, a focused on-farm dairy survey was conducted. The Nonambulatory Dairy On-Farm Questionnaire (available online at http://jds.fass.org/ content/vol91/issue6) was administered to a subsample of the NASS January 2005 Cattle Survey in 21 major dairy states representing $86 \%$ of the milk cow inventory and $84 \%$ of dairy operations in the United States (California, Colorado, Florida, Idaho, Illinois, Indiana, Iowa, Kentucky, Michigan, Minnesota, Missouri, New Mexico, New York, Ohio, Pennsylvania, Tennessee, Texas, Vermont, Virginia, Washington, Wisconsin). In the 21 states, all respondents that had 5 or more milk cows on January 1, 2005, and one or more nonambulatory milk cows or dairy calves in 2004 were selected ( $\mathrm{n}=$ 2,002 ), and a herd-size-based stratified random sample of respondents that had 5 or more milk cows on January 1,2005 , but did not have a nonambulatory milk cow or dairy calf in 2004 was selected for comparative purposes $(n=500)$. The control sample was weighted across herd- size categories ( 5 to 99,100 to 499 , and 500 or more milk cows) for each state, and designed to detect risk factors with estimated odds ratios in the range from 1.5 to 2.0 for operations having nonambulatory cattle. There was not a statistically significant difference in the predominant breed makeup of case herds vs. control herds.

During the period from April 1 to May 13, 2005, NASS enumerators administered a questionnaire on general management and facilities as well as the number, producer-specified primary causes, treatments, and outcomes of nonambulatory cattle and calves (although this was a NAHMS study, NAHMS contracted with NASS for questionnaire administration). The questionnaire was administered in person. Nonambulatory operations were defined as those that had one or more nonambulatory cows during 2004, including those that recovered. Nonambulatory cows were defined as those that were unable to stand or walk without assistance for any length of time, including those that recovered. Control operations were defined as those that had no nonambulatory cows during 2004.

Of the 2,002 case and 500 control operations, a total of 1,596 and 389 operations, respectively, responded to the questionnaire. The overall response rate was $79.3 \%$ $(1,985 / 2,502)$. Of the 389 responding control operations, 313 were reclassified as case operations based on responses to the NAHMS questionnaire. A smaller proportion of small and medium operations converted than did large operations. Of the 1,596 case operations, 75 became control operations upon additional questioning regarding nonambulatory cows. This result was counterintuitive. Possible explanations include different respondents for the telephone questionnaire vs. the onfarm questionnaire, and concern about participating in a study on nonambulatory dairy cattle when bovine spongiform encephalopathy had received recent publicity. The changing of case/control status resulted in 1,834 case operations and 151 control operations. Additionally, 12 case operations were not included in the analyses due to incomplete data.

Questionnaire data from the 1,822 nonambulatory operations and 151 control operations were used for descriptive and logistic regression analyses. Because of the small number of control operations, medium and large operations were combined for comparison with small operations for the operation-level analysis. The resulting 2 size groups were medium-to-large operations with 100 or more head and small operations with 5 to 99 head. Some variable categories were combined because of small numbers of operations having specific facility characteristics or utilizing a specific practice. For the sake of brevity, some questions that were asked 
are not reported here because the results were not different between case and control herds.

To get more detailed information about nonambulatory dairy cattle for the animal-level analysis, producers from case operations were asked to describe their most recent nonambulatory bovine $227 \mathrm{~kg}$ or more in 2004. Although producers had the opportunity to consult treatment records for these questions, the majority of producers responded based on recall. Recovery of the most recent nonambulatory bovine was defined as either a response of "recovered and remained in the herd" or "recovered and permanently removed from the herd." Other questionnaire responses were "permanently removed from the herd while unable to stand or walk"; "euthanized while on the operation"; "died"; and "other outcome." One animal per operation contributed to these estimates.

\section{Statistical Analysis}

Descriptive statistics were generated to describe herd-level and animal-level factors using statistical software that accounted for weighting to reflect the probability of selection and inclusion in the study. For the case operations, an initial selection weight of 1 was given, because a census of these operations was performed. For the control operations, the initial selection weight was equal to the inverse of probability of selection (SUDAAN Release 9.0.1, 2004; Research Triangle Institute, Research Triangle Park, NC). The CROSS$\mathrm{TAB}$ procedure was used to compare management and facilities characteristics between case and control operations, and to compare animal-level characteristics between nonambulatory cattle that recovered and those that did not. The PROC RLOGIST procedure was used to calculate odds ratios and $P$-values for association between operation-level factors and case-control status, as well as between animal-level factors and recovery status.

Facilities and management-related variables were analyzed individually for association with nonambulatory status while adjusting for herd size and accounting for study design and operation weights. Variables were considered for inclusion in the multivariable logistic regression model if the chi-square $P$-value $<0.25$. The variables for housing (indoor or outdoor), housing facility (free-stall, pen, multiple animal area, tie-stall, pasture, drylot, other), flooring surface (concrete, dirt, pasture, other), and flooring moisture (usually dry, wet about half the time, almost always wet but no standing water, usually standing water or slurry) were collinear. Thus, among these collinear variables, the one with the smallest $P$-value for each season (summer, winter) and cow class (dry, lactating) was selected for backward elimination logistic regression modeling. Managementrelated variables with an odds ratio of 2.0 or greater, and for which there was a sample size of 5 or greater for each level of the variable in each herd size category, were selected for backward elimination logistic regression modeling. Herd-size category was included in the model as a covariate. Using the Wald statistic, variables were removed from the model until those that remained were significant at $P<0.05$. Plausible interactions between variables that remained in the final model were examined.

To assess the fit of each model, sensitivity and specificity of the model-predicted outcomes were assessed by treating the observed status as the gold standard. A probability cut point of $\geq 0.5$ was used for predicted outcome; samples with a predicted probability of $\geq 50 \%$ that they were positive were classified as positive.

Animal-level variables were analyzed individually for association with recovery status while accounting for study design. Variables were considered for inclusion in the multivariable logistic regression model if the chisquare $P$-value $<0.25$. A backward elimination procedure was used and variables were removed from the model until those that remained had a Wald statistic $P$-value $<0.05$. Plausible interactions between variables that remained in the final model were examined.

\section{RESULTS}

\section{Comparison of Operations}

Overall, $30.7 \%$ of case operations and $7.3 \%$ of control operations had 100 or more cows (Table 1). More than half $(55.9 \%)$ of case operations and $28.1 \%$ of controls fed a TMR. Feeding anionic salts to springing heifers and bST use were not widely practiced. The rolling herd average was $9,090 \mathrm{~kg}$ or more on $52.3 \%$ of case operations and $20.1 \%$ of control operations. Footbaths were used on $31.3 \%$ of case operations and on $7.9 \%$ of control operations. Hoof trimming was performed on more than one-quarter of cows annually on $54.4 \%$ of case operations and $20.6 \%$ of control operations. A professional hoof trimmer (not a veterinarian or operation personnel) was used on $73.8 \%$ of case operations and $47.1 \%$ of control operations.

On $44.7 \%$ of case operations and $24.7 \%$ of control operations, the predominant flooring surface on which dry cows stood or walked in summer was concrete, dirt, or other (not pasture) (Table 2). On $65.7 \%$ of case operations and $42.4 \%$ of control operations, the predominant housing facility for lactating cows in summer was not pasture (included free-stall, pen, tie-stall, drylot, other). On $89.6 \%$ of case operations and $74.1 \%$ of control operations, the predominant housing facility for dry cows in winter was not pasture (included free-stall, pen, tie- 
Table 1. Percentage of nonambulatory case and control operations having the following management characteristics and herd-size adjusted odds ratios (OR), confidence intervals (CI) and $P$-values

\begin{tabular}{|c|c|c|c|c|c|c|c|}
\hline \multirow{2}{*}{$\begin{array}{l}\text { Management characteristics } \\
\text { (eligible for model entry) }\end{array}$} & \multicolumn{2}{|c|}{$\begin{array}{c}\text { Case } \\
\text { operations }\end{array}$} & \multicolumn{2}{|c|}{$\begin{array}{l}\text { Control } \\
\text { operations }\end{array}$} & \multirow{2}{*}{$\begin{array}{c}\text { Adjusted } \\
\text { univariate } \\
\text { OR } \\
\end{array}$} & \multirow{2}{*}{$\begin{array}{c}\text { Adjusted } \\
\text { univariate } \\
95 \% \text { CI }\end{array}$} & \multirow{2}{*}{$\begin{array}{c}\text { Adjusted } \\
\text { univariate } \\
P \text {-value }\end{array}$} \\
\hline & $\%$ & $\mathrm{SE}$ & $\%$ & $\mathrm{SE}$ & & & \\
\hline Medium to large herd size $(\geq 100 \text { cows })^{1}$ & 30.7 & $(1.0)$ & 7.3 & (1.4) & 5.6 & $3.3-9.6$ & $<0.01$ \\
\hline Fed TMR & 55.9 & $(1.9)$ & 28.1 & $(5.2)$ & 2.3 & $1.2-4.2$ & 0.01 \\
\hline Fed anionic salts to springing heifers ${ }^{2}$ & 13.8 & (1.5) & 3.5 & $(1.5)$ & 3.5 & $1.2-10.3$ & 0.03 \\
\hline Used any bST & 17.5 & (1.1) & 5.0 & $(0.9)$ & 2.6 & $1.4-5.0$ & $<0.01$ \\
\hline Rolling herd average $9,090 \mathrm{~kg}$ or more & 52.3 & (1.9) & 20.1 & $(3.5)$ & 3.5 & $2.0-5.9$ & $<0.01$ \\
\hline Used footbaths & 31.3 & (1.5) & 7.9 & $(2.0)$ & 3.2 & $1.7-6.0$ & $<0.01$ \\
\hline $\begin{array}{l}\text { Trimmed hooves on more than a quarter } \\
\text { of cows annually }\end{array}$ & 54.5 & $(1.9)$ & 20.6 & $(4.2)$ & 3.4 & $1.8-6.2$ & $<0.01$ \\
\hline $\begin{array}{l}\text { Used professional hoof trimmer } \\
\text { (not veterinarian or operation personnel) }\end{array}$ & 73.8 & (1.8) & 47.1 & $(7.4)$ & 2.5 & $1.2-4.9$ & 0.01 \\
\hline
\end{tabular}

stall, drylot, other). On $96.6 \%$ of case operations and $83.7 \%$ of control operations, the predominant flooring surface on which lactating cows stood or walked in winter was concrete, dirt, or other (not pasture).

In the final operation-level logistic regression analysis, operations with 100 or more cows were more likely to have nonambulatory cows compared with operations with 5 to 99 cows (Table 3). Operations that fed a TMR were more likely to have nonambulatory cows compared with those that did not. The interaction between herd size and feeding TMR was not significant. Operations with a rolling herd average of $9,090 \mathrm{~kg}$ or more were more likely to have nonambulatory cows compared with operations where the rolling herd average was less than $9,090 \mathrm{~kg}$. Operations where pasture was not the predominant flooring surface on which lactating cows stood or walked in winter were more likely to have nonambu- latory cows than operations where the predominant flooring surface was pasture. Sensitivity and specificity for the operation-level model were 98.9 and $4.8 \%$, respectively. Within this population, the positive predictive value for the model was $92.7 \%$ and the negative predictive value for the model was $26.9 \%$.

\section{Cow Recovery}

Overall, $10.1 \%$ of nonambulatory cows in their first lactation recovered, as did $17.7 \%$ in their second through fourth lactations, and $22.2 \%$ in their fifth or higher lactation. Of dry nonambulatory cows, $11.3 \%$ recovered, whereas $17.3 \%$ of lactating cows recovered. However, too few dry cows were present in the sample to be included in the multivariable analysis. Of cows that had received prior veterinary treatment, $11.6 \%$

Table 2. Percentage of nonambulatory case and control operations having the following facility characteristics and herd-size adjusted odds ratios (OR), confidence intervals $(\mathrm{CI})$ and $P$-values

\begin{tabular}{|c|c|c|c|c|c|c|c|}
\hline \multirow{2}{*}{$\begin{array}{l}\text { Facility characteristic } \\
\text { (eligible for model entry) }\end{array}$} & \multicolumn{2}{|c|}{$\begin{array}{c}\text { Case } \\
\text { operations }\end{array}$} & \multicolumn{2}{|c|}{$\begin{array}{c}\text { Control } \\
\text { operations }\end{array}$} & \multirow{2}{*}{$\begin{array}{c}\text { Adjusted } \\
\text { univariate } \\
\text { OR }\end{array}$} & \multirow{2}{*}{$\begin{array}{c}\text { Adjusted } \\
\text { univariate } \\
95 \% \text { CI }\end{array}$} & \multirow{2}{*}{$\begin{array}{c}\text { Adjusted } \\
\text { univariat } \\
P \text {-value }\end{array}$} \\
\hline & $\%$ & $\mathrm{SE}$ & $\%$ & $\mathrm{SE}$ & & & \\
\hline \multirow{4}{*}{$\begin{array}{l}\text { Predominant flooring on which dry cows stood } \\
\text { or walked in summer was concrete, dirt, or } \\
\text { other (not pasture) }^{1} \\
\text { Predominant housing facility for lactating cows } \\
\text { in summer was not pasture (includes free-stall, } \\
\text { pen, tie-stall, drylot, other) } \\
\text { Predominant housing facility for dry cows in winter } \\
\text { was not pasture (includes free-stall, pen, } \\
\text { tie-stall, drylot, other) } \\
\text { Predominant flooring on which lactating cows stood } \\
\text { or walked in winter was concrete, dirt, or other } \\
\text { (not pasture) }\end{array}$} & 44.7 & (1.9) & 24.7 & (5.1) & $1.9^{2}$ & $1.0-3.6$ & 0.04 \\
\hline & 65.7 & (1.9) & 42.4 & (4.9) & 2.0 & $1.2-3.3$ & $<0.01$ \\
\hline & 89.6 & (0.9) & 74.1 & $(4.0)$ & 3.4 & $1.9-5.9$ & $<0.01$ \\
\hline & 96.6 & $(0.5)$ & 83.7 & (3.7) & 6.8 & $3.3-14.0$ & $<0.01$ \\
\hline
\end{tabular}

${ }^{1}$ More than $50 \%$ of operations reported concrete as the predominant flooring surface.

${ }^{2}$ Corresponds to smallest $P$-value for facilities and flooring-related variables for dry cows in summer. 
Table 3. Results of herd-level backward elimination logistic regression model for herds having nonambulatory cows

\begin{tabular}{lccr}
\hline Herd characteristics (eligible for model entry) & $\begin{array}{c}\text { Odds } \\
\text { ratio }\end{array}$ & $\begin{array}{c}\text { Confidence } \\
\text { interval }\end{array}$ & $P$-value \\
\hline Medium to large herd size (100 or more cows) & 3.7 & $2.2-6.2$ & $<0.01$ \\
$\begin{array}{l}\text { Fed TMR } \\
\text { Rolling herd average 9,090 kg or more }\end{array}$ & 2.0 & $1.1-3.4$ & 0.02 \\
$\begin{array}{l}\text { Predominant flooring surface on which lactating cows } \\
\text { stood or walked in winter was concrete, dirt, or other }\end{array}$ & 2.8 & $1.8-4.5$ & $<0.01$ \\
(not pasture) $^{1}$ & & & $<0.01$ \\
\hline
\end{tabular}

${ }^{1}$ More than $50 \%$ of these operations had concrete as the predominant flooring surface.

recovered, whereas of cows that had not received prior treatment, $18.0 \%$ recovered. Of cows with a BCS $<2.5$, $8.1 \%$ recovered, and of cows with a BCS of $\geq 2.5,16.6 \%$ recovered. Of cows $<15 \mathrm{~d}$ in milk, $28.4 \%$ recovered, and of cows $\geq 15$ days in milk, $6.2 \%$ recovered. Of cows that were nonambulatory for $<24 \mathrm{~h}, 32.9 \%$ recovered. Of cows that were nonambulatory for $\geq 24 \mathrm{~h}, 8.2 \%$ recovered. Of nonambulatory cows from operations of fewer than 100 cows, $18.4 \%$ recovered, of cows from operations of 100 to 499 cows, $10.9 \%$ recovered, and of cows from operations of 500 or more cows, $9.3 \%$ recovered (Table 4).

Of cows that were repositioned, $9.4 \%$ recovered. Of cows that were not repositioned, $27.5 \%$ recovered. Of cows that received calcium, phosphorus, or potassium, $27.6 \%$ recovered, and of cows that did not, $6.6 \%$ recovered. Of cows that received an assisted rise, $11.1 \%$ recovered, and of cows that did not, $18.7 \%$ recovered (Table 5).

In the final animal-level logistic regression analysis, cows nonambulatory for $<24 \mathrm{~h}$ were more likely to recover compared with cattle nonambulatory for $\geq 24 \mathrm{~h}$ (Table 6). Cows that received calcium, phosphorus, or potassium while nonambulatory were more likely to recover compared with those that did not receive calcium, phosphorus, or potassium. Cows that were not repositioned periodically were more likely to recover compared with cattle that were repositioned. Cows that were not treated by a veterinarian before becoming nonambulatory were more likely to recover than those that were treated prior to becoming nonambulatory. In comparison with the "other causes" reference group (cancer, clinical mastitis, digestive condition, metabolic imbalance, neurological problems, respiratory disease, other, unknown), nonambulatory cows with hypocalcemia were more likely to recover. There was no significant difference in the likelihood of recovery between cows that became nonambulatory due to calving-related injury and those that became nonambulatory due to other causes. Similarly, the difference in likelihood of recovery between cows that became nonambulatory because of lameness, slipping, or falling versus other causes was not significant. Herd size did not remain in the final model. The interaction between length of time nonambulatory and repositioning was not significant. Sensitivity and specificity for the cow-level model were 39.5 and $98.9 \%$, respectively. Within this population, the positive predictive value for the model was $83.8 \%$ and the negative predictive value for the model was $91.8 \%$ (Table 6).

\section{DISCUSSION}

Causes of nonambulatory cattle can be divided into general categories including injuries, metabolic imbalances, and infectious or toxic disease. In the veterinary literature, a variety of case definitions for nonambulatory cattle have been utilized, some of which include parameters for time spent nonambulatory or outcome (e.g., recovery vs. death/euthanasia). Hansen and Bridges (1999) provided recovery estimates for nonambulatory cattle with neurologic signs of unknown etiology. In that study, progressive neurologic signs were defined to include gradual paralysis, behavior changes, abnormal gait, or other recognizable central nervous system (CNS) signs before death. The recovery rate for nonambulatory dairy cows with nonprogressive CNS signs was $26 \%$. Of the progressive CNS cases in the study, 29\% recovered. Trauma/injury, septicemia/toxemia, and responsive milk fever cases accounted for $85 \%$ of the nonambulatory nonprogressive cows; known and unknown CNS conditions, septicemia/toxemia, and nonresponsive milk fever cases accounted for $75 \%$ of the nonambulatory progressive cases.

Cox et al. (1986) surveyed producers about nonambulatory cattle in Minnesota dairy herds. In this study, cows were classified as nonambulatory if they had been recumbent on the sternum for more than $24 \mathrm{~h}$, and did not die within $3 \mathrm{~d}$ of becoming nonambulatory. By this definition, an estimated $2.1 \%$ of cows in the surveyed Minnesota dairy herds became nonambulatory in 1983. Of these, 33\% recovered, $23 \%$ were slaughtered, and 
Table 4. Percentage of nonambulatory cattle that recovered, univariate odds ratios (OR), and $P$-values by animal and herd size characteristics

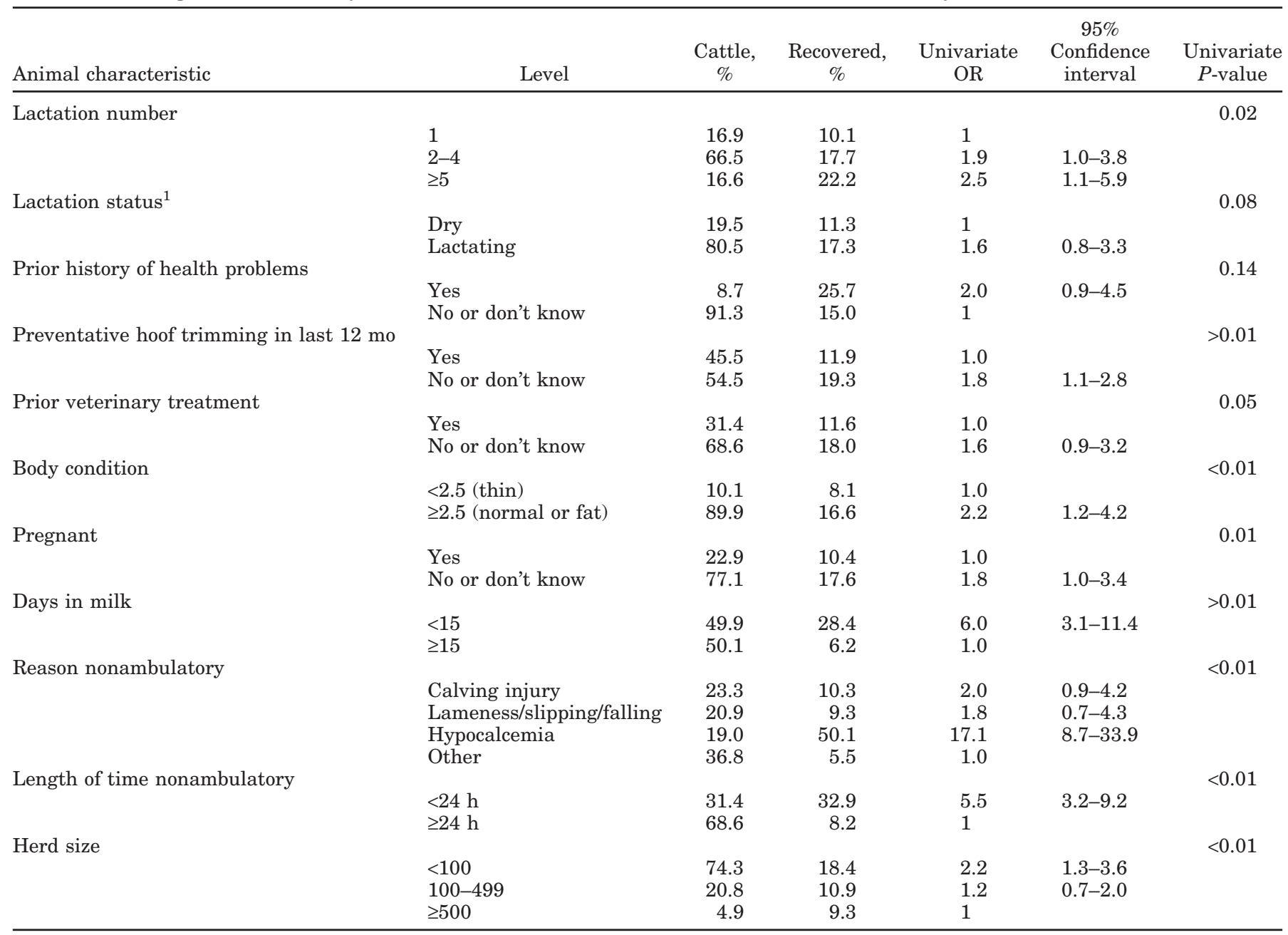

${ }^{1}$ Too few dry cows in sample to include variable in model.

Table 5. Percentage of nonambulatory cattle that recovered, univariate odds ratios (OR), and $P$-values, by treatment characteristic

\begin{tabular}{lcccccc}
\hline Treatment characteristic & Level & $\begin{array}{c}\text { Cattle, } \\
\%\end{array}$ & $\begin{array}{c}\text { Recovered, } \\
\%\end{array}$ & $\begin{array}{c}\text { Univariate } \\
\text { OR }\end{array}$ & $\begin{array}{c}\text { Confidence } \\
\text { interval }\end{array}$ & $\begin{array}{c}\text { Univariate } \\
P \text {-value }\end{array}$ \\
\hline Repositioned & Yes & 64.2 & 9.4 & 1 & & $<0.01$ \\
& No & 35.8 & 27.5 & 3.7 & $2.2-6.0$ & $<0.01$ \\
Received Ca, P, or K & Yes & 44.2 & 27.6 & 5.4 & $3.2-9.1$ & $<0.01$ \\
& No & 55.8 & 6.6 & 1 & & $<0.01$ \\
Received antibiotic & Yes & 35.3 & 10.6 & 1 & & \\
& No & 64.7 & 18.9 & 2.0 & $1.2-3.3$ & \\
Received anti-inflammatory & Yes & 35.5 & 10.4 & 1 & & $<0.01$ \\
& No & 64.5 & 19.0 & 2.0 & $1.2-3.3$ & \\
Assisted rise & Yes & 36.3 & 11.1 & 1 & & \\
& No & 63.7 & 18.7 & 1.8 & $1.1-3.2$ & \\
\hline
\end{tabular}


Table 6. Results of cow-level backward elimination logistic regression model for cows recovering from a nonambulatory condition

\begin{tabular}{|c|c|c|c|c|}
\hline Factor & Level & Odds ratio & $\begin{array}{l}\text { 95\% Confidence } \\
\text { interval }\end{array}$ & $P$-value \\
\hline \multirow[t]{3}{*}{ Prior veterinary treatment } & & & & 0.02 \\
\hline & Yes & 1.0 & & \multirow{7}{*}{$<0.01$} \\
\hline & No & 1.9 & $1.1-3.3$ & \\
\hline \multirow[t]{5}{*}{ Reason nonambulatory } & & & & \\
\hline & Calving injury & 1.8 & $0.9-3.8$ & \\
\hline & Lameness/slipping/falling & 2.1 & $1.0-4.8$ & \\
\hline & Hypocalcemia & 6.0 & $3.4-10.7$ & \\
\hline & Other & 1.0 & & \\
\hline \multirow[t]{3}{*}{ Length of time nonambulatory } & & & & \multirow[t]{3}{*}{$<0.01$} \\
\hline & $<24 \mathrm{~h}$ & 3.0 & $2.0-4.4$ & \\
\hline & $24 \mathrm{~h}+$ & 1 & & \\
\hline \multirow{2}{*}{ Repositioned } & Yes & 1.0 & & \multirow{2}{*}{$<0.01$} \\
\hline & No & 2.1 & $1.4-3.1$ & \\
\hline \multirow{3}{*}{ Received $\mathrm{Ca}, \mathrm{P}$, or $\mathrm{K}$} & & & & \multirow[t]{3}{*}{$<0.01$} \\
\hline & Yes & 3.6 & $2.1-6.1$ & \\
\hline & No & 1 & & \\
\hline
\end{tabular}

$44 \%$ died or were killed. Calving difficulty was the primary cause of $24 \%$ of nonambulatory cows, although $58 \%$ of the cows became nonambulatory within a day of calving. An additional $37 \%$ became nonambulatory within the first $100 \mathrm{~d}$ of lactation. Forty-eight percent of nonambulatory cows were classified by owners as high producers. Many other studies focus mainly on cattle that were nonambulatory due to milk fever (Dohoo et al., 1983, 1984; Dohoo and Martin, 1984).

\section{Herd Level}

The farm-level estimate that $78.2 \%$ of operations in 21 dairy states had one or more nonambulatory cows in 2004 (Green et al., 2006) is higher than that reported by NASS in 2004 (26.5\%; NASS, 2005). There are several reasons for this difference. One is that the NASS estimates reflect all dairy operations with one or more cows, whereas the NAHMS study estimates were based on data collection from dairy operations with 5 or more dairy cows in 21 major dairy states. Another is that during the NAHMS interview process, the definition of nonambulatory was discussed in detail with producers, which may have helped the producers better recall nonambulatory cows that recovered.

In the final multivariable model, the increased likelihood of having nonambulatory cows that was associated with a herd size of 100 or more cows was expected, given that having a larger number of cows presents a greater number of opportunities for events leading to a nonambulatory condition. The result that feeding a TMR was associated with an increased likelihood of having nonambulatory cows was unexpected. Use of a TMR feeding system is generally considered to improve cow health compared with traditional component feed- ing. With a properly mixed and formulated TMR, there is little variation in nutrient content; therefore, the rumen environment remains stable and the risk of acidosis is minimized. However, the questionnaire did not request additional information that might have provided insight into potential problems with TMR formulation, such as whether forage testing was utilized, and whether dry matter content and particle size were monitored. Information on how much TMR was consumed, types of mixing systems utilized, whether the TMR contained all portions of the ration, or whether hay was fed separately could account, in part, for increased odds of having nonambulatory cattle. Information on other feeding systems (component feeding, management-intensive grazing) was not collected.

One possible explanation for this result is that feeding a TMR acts as a proxy for several associated management factors. Of the operations that utilized bST, 93.6\% fed a TMR, compared with $61.8 \%$ of operations that did not utilize bST. Over $80 \%$ of lactating cows (83.9\%) and dry cows $(81.9 \%)$ that were on flooring surfaces that were wet half the time or more during winter were fed a TMR, whereas $61.4 \%$ of lactating cows and $62.8 \%$ of dry cows that were on flooring surfaces that were usually dry during winter were fed a TMR (data not presented). The relationship between feeding a TMR and flooring-surface moisture in summer was similar for both lactating and dry cows.

The finding that a high rolling herd average milk production was associated with an increased likelihood of having nonambulatory cows is consistent with the literature on the challenges of implementing management practices associated with high milk production. High-producing dairy herds faced with maximizing energy intake while maintaining the health of the lactat- 
ing cow may be challenged with subclinical acidosis and laminitis (Nocek, 1997; Collard et al., 2000), both of which may ultimately lead to nonambulatory cattle.

Concrete flooring has been implicated as a risk factor for dairy cow claw disorders such as digital dermatitis (Wells et al., 1999; Somers et al., 2003) and heel horn erosions (Sogstad et al., 2005). There is also evidence that cows on concrete flooring have difficulty standing up and lying down (Haley et al., 2001). Previous studies have shown that cows that spend the majority of time on concrete may have increased problems with lameness (Somers et al., 2003) because of concrete's abrasive properties. Therefore, it seems plausible that concrete flooring could be associated with lameness or injury that may ultimately result in nonambulatory cows.

A model sensitivity of $98.9 \%$ indicates that nearly 99\% of the operations that had a nonambulatory cow would be correctly classified as nonambulatory operations by the model. A specificity of $4.8 \%$ indicates that less than $5 \%$ of operations with no nonambulatory cattle would be correctly identified. Thus, the model was heavily biased in terms of effectively predicting operations that had nonambulatory cattle.

Because the nonambulatory state can result from many metabolic, infectious, and injury-related processes, it was not unexpected that several factors are significantly associated with an operation having nonambulatory cattle. Additional studies on the specific reasons cattle become nonambulatory are likely to provide more focused insight.

\section{Cow Level}

The greater rate of recovery for nonambulatory cows of 5 lactations or more, cows less than $15 \mathrm{~d}$ in milk, cows from small herds, and cows that received calcium, phosphorus, or potassium in the univariate analyses are all most likely related to hypocalcemia. An explanation for the apparent lack of benefit of repositioning, anti-inflammatory drugs, and assisted rises is that these treatments are most likely undertaken when a cow has already been nonambulatory for an extended period. Another possibility is that more serious cases may be more likely to receive aggressive treatment.

In the final multivariable model for cow recovery, the finding that cows with a history of treatment by a veterinarian before becoming nonambulatory were less likely to recover than those that had no such history is compatible with a poor prognosis if a history of chronic health problems was present. A thorough physical exam and history before pursuing treatment should assist in minimizing costs and animal suffering (Stull et al., 2007).
Cows that were nonambulatory for less than $24 \mathrm{~h}$ were more likely to recover compared with cattle nonambulatory for $24 \mathrm{~h}$ or more. As cattle remain nonambulatory, the muscle and nerve damage that occurs as a result can become more severe, further decreasing the likelihood of recovery (Cox, 1982, 1988). It has been suggested that $6 \mathrm{~h}$ is the time threshold for secondary recumbency, after which time, following correction of the initial reason for recumbency, pressure-related muscle and nerve damage that prevent recovery have occurred (Cox, 1982; Fenwick et al., 1986). Therefore, nonambulatory cattle should be treated as medical emergencies. A timely decision on whether to pursue treatment should be made considering likelihood of successful outcome, cost, and duration of pain and suffering. If treatment is not pursued, then humane euthanasia should not be delayed (Stull et al., 2007).

Although cows that were repositioned were less likely to recover than those that were not, this association should not be interpreted as a negative effect of repositioning. For example, cows with hypocalcemia may respond to calcium therapy within a few hours of being nonambulatory and not necessitate repositioning, other than for the ease of providing intravenous therapy. Certainly, quality of care affects prognosis (Cox, 1988), and it is likely that repositioning is generally initiated when it becomes clear that an animal may be nonambulatory for a prolonged period and precautions to prevent additional nerve and muscle damage are needed. Recumbent cows should be rolled every several hours, alternating between the left and right sides, to prevent secondary muscle and nerve damage (Kronfeld, 1976; Jardon, 1993). Nonambulatory cattle should be examined daily to determine any change in ability to rise or bear weight (Stull et al., 2007).

The findings that hypocalcemia as a cause of the nonambulatory state and treatment with calcium, phosphorus, or potassium were associated with an increased likelihood of recovery are consistent with hypocalcemia as one of the most treatable causes of nonambulatory cows.

A model sensitivity of $39.5 \%$ indicates that the model correctly predicts about $40 \%$ of the nonambulatory cows that recovered. A specificity of $98.9 \%$ indicates that nearly $100 \%$ of cows that did not recover would be correctly identified. Thus, the model is much more effective in prediction of cows that did not recover from the nonambulatory state. A limitation of this study was that reporting of nonambulatory cattle relied upon retrospective assessments of disease occurrence by herd managers, and some cases of disease are likely to be forgotten by producers. This reporting bias could influence analysis if it changed the nonambulatory status of the herd. 


\section{CONCLUSIONS}

The results of this study reveal that the majority of US dairy operations have at least one nonambulatory dairy cow over the course of a year. Additionally, individual-animal factors associated with being nonambulatory may lead to improved treatment and assessment of the likelihood for recovery for cattle that are nonambulatory for a prolonged period of time. From the perspectives of recovery and welfare, euthanasia should be considered for cows that have been nonambulatory for more than $24 \mathrm{~h}$.

\section{REFERENCES}

Collard, B. L., P. J. Boettcher, J. C. Dekkers, D. Petitclerc, and L. R. Schaeffer. 2000. Relationships between energy balance and health traits of dairy cattle in early lactation. J. Dairy Sci. 83:2683-2690.

Cox, V. S. 1982. Pathogenesis of the downer cow syndrome. Vet. Rec. 111:76-79.

Cox, V. S. 1988. Nonsystemic causes of the downer cow syndrome. Vet. Clin. North Am. Food Anim. Pract. 4:413-433.

Cox, V. S., W. E. Marsh, G. R. Steurenagel, T. F. Fletcher, and J. S. Onapito. 1986. Downer cow occurrence in Minnesota dairy herds. Prev. Vet. Med. 4:249-260.

Department for Environment, Food, and Rural Affairs (Defra). 2003. Code of Recommendations for the Welfare of Livestock-Cattle http://www.defra.gov.uk/animalh/welfare/farmed/cattle/ booklets/cattcode.pdf Accessed Jun. 4, 2007.

Dieterlen, P. L., and J. A. Strasser. 2006. Cooperative Agreement \#FSIS-C-27-2002 Development of an inspection-based quality assurance and certification program for market cattle and swine. Indiana Board of Animal Health, Indianapolis.

Dohoo, I. R., and S. W. Martin. 1984. Disease, production, and culling in Holstein-Friesian cows II. Age, season and sire effects. Prev. Vet. Med. 2:655-670.

Dohoo, I. R., S. W. Martin, and A. H. Meek. 1984. Disease, production, and culling in Holstein-Friesian cows VI. Effects of management on disease rates. Prev. Vet. Med. 3:15-28.
Dohoo, I. R., S. W. Martin, A. H. Meek, and W. D. D. Sandals. 1983. Disease, production and culling in Holstein-Friesian cows: I. The data. Prev. Vet. Med. 1:321-334.

Fenwick, D. C., W. R. Kelly, and R. C. W. Daniel. 1986. Definition of non-alert downer cow syndrome and some case histories. Vet. Rec. 118:124-128.

Government of Canada. 2004. Health of Animals Regulations. Section 2 . http://canadagazette.gc.ca/partI/2004/20041218/html/regle3e.html\#1 Accessed Jun. 5, 2007.

Green, A., G. Hill, J. Lombard, L. Garber, B. Wagner, M. Parker, D. Dargatz, S. Bruntz, and N. Wineland. 2006. Factors associated with nonambulatory cattle on U.S. dairy operations. Proc. 11th Symp. Int. Soc. Vet. Epidemiol. Econ., Cairns, Australia. ISVEE XI Committee; abstract 817.

Haley, D. B., A. M. de Passille, and J. Rushen. 2001. Assessing cow comfort: Effects of two floor types and two tie stall designs on the behavior of lactating dairy cows. Appl. Anim. Behav. Sci. 71:105-117.

Hansen, D., and V. Bridges. 1999. A survey description of down-cows and cows with progressive or non-progressive neurological signs compatible with a TSE from veterinary-client herds in 38 states. Bovine Pract. 33:179-187.

Jardon, P. 1993. Care of downer cows. CA Dairy Herd Improvement Assoc., Feb:8-10. CA DHIA, Clovis.

Kronfeld, D. S. 1976. Management of downer cows. Mod. Vet. Pract. 57:599-602.

NASS. 2005. Non-Ambulatory Cattle and Calves. http://usda.mannlib.cornell.edu/usda/current/nacac/nacac-05-05-2005.pdf Accessed July 9, 2007.

NASS. 2006. NASS Land in Farms, and Livestock Operations. http:// usda.mannlib.cornell.edu/usda/nass/FarmLandIn//2000s/2006/ FarmLandIn-01-31-2006.pdf Accessed July 10, 2007.

Nocek, J. E. 1997. Bovine acidosis: Implications on laminitis. J. Dairy Sci. 80:1005-1028.

Sogstad, A. M., T. Fjeldaas, and O. Osteras. 2005. Lameness and claw lesions of the Norwegian Red dairy cattle housed in free stalls in relation to environment, parity and stage of lactation. Acta Vet. Scand. 46:203-217.

Somers, J. G. C. J., K. Frankena, E. N. Noordhuizen-Stassen, and J. H. M. Metz. 2003. Prevalence of claw disorders in Dutch dairy cows exposed to several floor systems. J. Dairy Sci. 86:2082-2093.

Stull, C. L., M. A. Payne, S. L. Berry, and J. P. Reynolds. 2007. Welfare of non-ambulatory cattle in the U.S. J. Am. Vet. Med. Assoc. 231:227-234.

Wells, S. J., L. P. Garber, and B. A. Wagner. 1999. Papillomatous digital dermatitis and associated risk factors in US dairy herds. Prev. Vet. Med. 38:11-24. 